\title{
A Combination of Scutellaria Baicalensis and Acacia Catechu Extracts for Short-Term Symptomatic Relief of Joint Discomfort Associated with Osteoarthritis of the Knee
}

\author{
Bahram H. Arjmandi,,2 Lauren T. Ormsbee, ${ }^{1,2}$ Marcus L. Elam, ${ }^{1,2}$ Sara C. Campbell, ${ }^{3}$ \\ Nader Rahnama, ${ }^{1,2,4}$ Mark E. Payton, ${ }^{5}$ Ken Brummel-Smith, ${ }^{6}$ and Bruce P. Daggy, ${ }^{1,2}$ \\ ${ }^{1}$ Department of Nutrition, Food, and Exercise Sciences, ${ }^{2}$ Center for Advancing Exercise and Nutrition Research on Aging, \\ and ${ }^{6}$ Department of Geriatrics, College of Medicine, the Florida State University, Tallahassee, Florida, USA. \\ ${ }^{3}$ Department of Exercise Science and Sport Studies, Rutgers University, the State University of New Jersey, \\ New Brunswick, New Jersey, USA. \\ ${ }^{4}$ Faculty of Sports Sciences, University of Isfahan, Isfahan, Iran. \\ ${ }^{5}$ Department of Statistics, Oklahoma State University, Stillwater, Oklahoma, USA.
}

\begin{abstract}
The extracts of Scutellaria baicalensis and Acacia catechu have been shown in previous studies to alleviate joint discomfort, reduce stiffness, and improve mobility by reducing the production of proinflammatory molecules over long periods of supplementation. The acute effects of intake of these extracts have not yet been investigated. Thus, we carried out a 1 week clinical trial to examine the extent to which UP446- a natural proprietary blend of S. baicalensis and A. catechu (UP446) — decreases knee joint pain, mobility, and biomarkers of inflammation in comparison to naproxen. Seventy-nine men and women (40-90 years old) diagnosed as having mild to moderate osteoarthritis (OA) consumed either 500 mg/day of the UP446 supplement or $440 \mathrm{mg} /$ day of naproxen for 1 week in a double-blind randomized control trial. Pain, knee range of motion (ROM), and overall physical activity were evaluated at the start and at the end of treatment. Fasting blood was collected to determine serum interleukins $1 \beta$ and 6 , tumor necrosis factor- $\alpha$, C-reactive protein, and hyaluronic acid. The UP446 group experienced a significant decrease in perceived pain $(P=.009)$ time dependently. Stiffness was significantly reduced by both treatments ( $P=.002$ UP446, $P=.008$ naproxen). Significant increases in mean ROM over time $(P=.04)$ were found in the UP446 group. These findings suggest that UP446 is effective in reducing the physical symptoms associated with knee OA.
\end{abstract}

KEY WORDS: • flavonoids $\bullet$ herbal supplement $\bullet$ inflammation $\bullet$ joint mobility $\bullet$ knee pain $\bullet$ osteoarthritis $\bullet$ pain

\section{INTRODUCTION}

$\mathbf{O}$ STEOARTHRITIS (OA) IS THE MOST COMMON joint disorder and a major contributor to disability for the elderly in the United States. ${ }^{1}$ Although there is a lack of agreement regarding the definition of $\mathrm{OA}$, it is generally viewed as a degenerative disorder involving cartilage degradation, resulting in pain, swelling, and loss of joint mobility. Several conventional treatments-most commonly, prescribed pharmaceuticals - are available to those suffering from OA, but compliance is usually low and often associated with side effects. Therefore, there is still a need to find safer and more effective natural remedies for alleviating and attenuating OA symptoms.

Among the therapeutic options, alternative and complementary approaches such as the use of a wide array of nat-

Manuscript received 15 January 2013. Revision accepted 10 December 2013.

Address correspondence to: Bahram H. Arjmandi, PhD, RD, Department of Nutrition, Food, and Exercise Sciences, 436 Sandels Building, Florida State University, Tallahassee, FL 32306, USA, E-mail: barjmandi@fsu.edu ural, herbal, nutritional, holistic, and physical manipulations have become popular. ${ }^{2}$ For instance, dietary supplements containing chondroitin/glucosamine sulfate have been well received by the general public and have shown efficacy against the symptoms of OA. ${ }^{3}$

Flavonoids, in particular isoflavones, exert beneficial effects on cartilage metabolism. ${ }^{4,5}$ Flavonoids can attenuate inflammation ${ }^{6,7}$ and the immune response via the inhibition of important regulatory enzymes involved in arachidonic acid metabolism. ${ }^{8}$ Lin et al. ${ }^{9}$ demonstrated that flavonoids are able to inhibit the expression and activation of the proinflammatory cytokines interleukins $-1 \beta$ (IL-1 $\beta$ ) and -6 (IL-6), tumor necrosis factor $\alpha$ (TNF- $\alpha$ ), and cyclooxygenase 2 (COX-2), which is a known regulator of the production of inflammatory eicosanoids. In the same investigation, flavonoids downregulated matrix metalloproteinases (MMP), enzymes involved in the process of cartilage degradation, while upregulating MMP inhibitor in human synovial fibroblasts. Thus, flavonoids have the capacity to improve OA conditions via the reduction of cytokine activity. 
Generally, COX-2 inhibitors and similar NSAID drugs are considered efficacious for treating pain and functional impairment propagated by OA. ${ }^{1,10}$ Naproxen is a wellknown and effective drug for reducing the inflammatory response that is associated with OA, though the occurrence of side effects may pose compliance issues. ${ }^{11}$ Naproxen has been commonly used as a positive control for determining the magnitude of effect of other drugs and supplements, ${ }^{2,12-14}$ and we chose to do the same for the present study.

A medical food formulation composed of a concentrated ( $>90 \%$ purity) combination of the two botanical extracts Scutellaria baicalensis and Acacia catechu, known as flavocoxid, has been shown to be safe ${ }^{15}$ and effective in alleviating joint discomfort, reducing stiffness, and improving mobility. ${ }^{16,17}$ The content of flavonoids in the dietary supplement UP446 is approximately 85:15 Free-B-ring flavonoids to flavans. The Free-B-ring flavonoids are of Scutellaria origin and the flavans of Acacia origin. Flavocoxid is not available to the public without a prescription. Thus, UP446 has been developed for use as a dietary supplement. The efficacy of such proprietary blends is largely attributed to the inhibition of COX-2 and lipoxygenase (LOX) activities, ${ }^{15,18}$ both of which regulate the generation of proinflammatory molecules. The previous studies lasted for 4-12 weeks and did not examine whether the extracts had any effect in periods of less than 1 month. In the present study, we investigated the short-term effects of a proprietary dietary supplement known as UP446 for the alleviation of pain associated with $\mathrm{OA}$ and for the improvement of knee joint mobility. In terms of safety, UP446 has been extensively tested in animals ${ }^{19}$ and used in a randomized clinical trial. ${ }^{20}$ Here, we provide evidence that UP446 has beneficial effects for knee OA-associated symptoms, analogous to that of naproxen.

\section{MATERIALS AND METHODS}

\section{Study population}

A total of 252 otherwise healthy, overweight or obese men and women aged 40-90 years old with clinically diagnosed mild to moderate bilateral or unilateral $\mathrm{OA}$ of the knee and a Kellgren-Lawrence radiological score of 1-3 were screened and recruited from the greater Tallahassee, Florida, and surrounding areas using flyers and radio and online listings. Exclusion criteria included a history of severe liver and/or kidney disease or any other chronic or acute diseases that affect OA, any allergy or reaction to shellfish or naproxen, knee surgery (including arthroscopy) or significant injury of the target knee joint within 6 months prior to the start of the study, as well as any hyaluronan or cortisone injections within 2 months prior to study enrollment. The Institutional Review Board (IRB) at Florida State University approved the study protocol (no. 2009.3380), consent form, and all participant-related materials, and guidelines were strictly followed. After the initial prescreening over the telephone, eligible subjects were invited to the Human Performance Laboratory of FSU's Department of Nutrition, Food, and Exercise Sciences for the final screening. During this time, written informed consent and a complete medical history and 3 day nutrition log were obtained. A complete blood count was performed to exclude subjects further and confirm pre-existing conditions. Inclusion and exclusion criteria allowed 79 individuals to participate in the study. Those enrolled were able to walk unassisted and had experienced knee pain for at least 6 months.

Eligible subjects were also required to refrain from initiating and/or discontinue the use of any medications or supplements known to influence OA pain during the course of the study. Subjects taking any additional supplements or prescription drugs for the treatment of OA underwent a 2 week washout period prior to baseline.

\section{Experimental design}

Subjects were randomly assigned from a pregenerated randomization list to receive either the UP446 supplement containing extracts of $S$. baicalensis and A. catechu $(500 \mathrm{mg} /$ day $)$ or a control-naproxen sodium $(440 \mathrm{mg} /$ day)-for 1 week in a double-blinded manner in which treatment capsules were indistinguishable from one another. On baseline and final test days, each subject underwent basic anthropometric (height, weight, body mass index [BMI]) and vital sign assessments, (heart rate and blood pressure), followed by a venous blood draw, physical activity and range of motion (ROM) assessments, and a 6-minute walk test (6MWT). The Western Ontario and McMaster Universities Arthritis Index (WOMAC) pain assessment was completed once every 2 days throughout the 1 week trial.

\section{Western Ontario and McMaster Universities Arthritis Index and physical activity assessments}

The WOMAC assessment form was designed to measure lower extremity pain and function in $\mathrm{OA}$ of the knee or hip. ${ }^{21}$ Subjects reported subscale scores from 0 (mild) to 4 (extreme) for pain, stiffness, and difficulty in performing basic physical tasks during the previous 48 hours. A total score $(0-100)$ was then derived from the tallying of subscale scores.

The Five-City Project Physical Activity Recall ${ }^{22}$ was used to assess recent sleep, overall pattern of activity, and level of physical activity from baseline to final. The level of physical activity was based on either moderate (3-5 metabolic equivalents [METs], e.g., mopping and brisk walking), hard (5.1-6.9 METs, e.g., scrubbing floors and jogging), or very hard activities $(\geq 7.0$ METs, e.g., cross-country skiing and high-impact aerobics).

\section{6-minute walk and range of motion tests}

A 6MWT was completed by each subject based on a standardized protocol ${ }^{23}$ at baseline and final. The subjects were instructed to walk as quickly as possible without pain or discomfort for 6 minutes on a flat surface while wearing comfortable shoes. During testing, research personnel refrained from walking alongside or in front of the subjects to 
avoid influencing their pace. The total distance covered (meters) was measured using a trundle wheel.

Changes in knee $\mathrm{ROM}\left({ }^{\circ}\right)$ were assessed using a computerized isokinetic dynamometer (Biodex, Shirley, NY, USA) at baseline and final. The leg most affected by symptoms associated with OA was used for ROM testing. Subjects were seated in an upright position with the test leg secured at $90^{\circ}$, and the dynamometer arm rotor aligned to the lateral side of the knee joint. Each subject was instructed to raise the shank of the leg as high as he/she could for a period (approximately $2 \mathrm{sec}$ ) long enough for a steady ROM value to be reached. Three testing bouts were performed, and an average ROM was recorded.

\section{Blood collection and assays}

Fasting ( $\geq 8 \mathrm{~h}$ ) blood samples were collected between the hours of 5:00AM and 10:00AM on baseline and final days of the study. Blood was centrifuged at $2500 \mathrm{~g}$ and $4^{\circ} \mathrm{C}$ for $15 \mathrm{~min}$, after which serum was collected and stored at $-80^{\circ} \mathrm{C}$ until analyzed. Serum concentrations of C-reactive protein (CRP), IL-1 $\beta$ and IL-6 TNF- $\alpha$, all markers of inflammation, and hyaluronic acid (HA), a marker of cartilage degradation, were determined for baseline and final samples. Serum CRP concentrations were determined using commercially available high-sensitivity CRP ELISA kits (IBL International, Hamburg, Germany). Pro-inflammatory cytokines
IL-1 $\beta$, IL-6, and TNF were determined using BD OptEIA ${ }^{\mathrm{TM}}$ ELISA kits purchased from BD Biosciences (San Jose, CA, USA). Serum HA concentrations were also determined using commercially available ELISA kits (Echleon Biosciences, Salt Lake City, UT, USA). Each assay sample was run in duplicate.

\section{Statistical analyses}

All statistical analyses were conducted with SAS v9.2 (SAS Institute, Inc., Cary, NC, USA). Analysis of variance (ANOVA) techniques were used to assess the effects of treatment and time. A two-factor model with repeated measures was assumed. The intrasubject correlation was modeled with an autoregressive period 1 covariance. Simple effects of treatment given time, and time given treatment, were assessed with planned orthogonal contrasts. All data are presented as mean \pm standard error.

\section{RESULTS}

\section{Subject characteristics}

Baseline values, including age, weight, BMI, heart rate, and blood pressure of all subjects in this study are presented in Table 1. All baseline values for age, weight, and BMI were similar between the treatment groups. Figure 1 shows 84 subjects randomly assigned to receive UP446 or naproxen.

Table 1. Subject Characteristics, Physical Activity, and Functional Changes: Group and Time Interactions

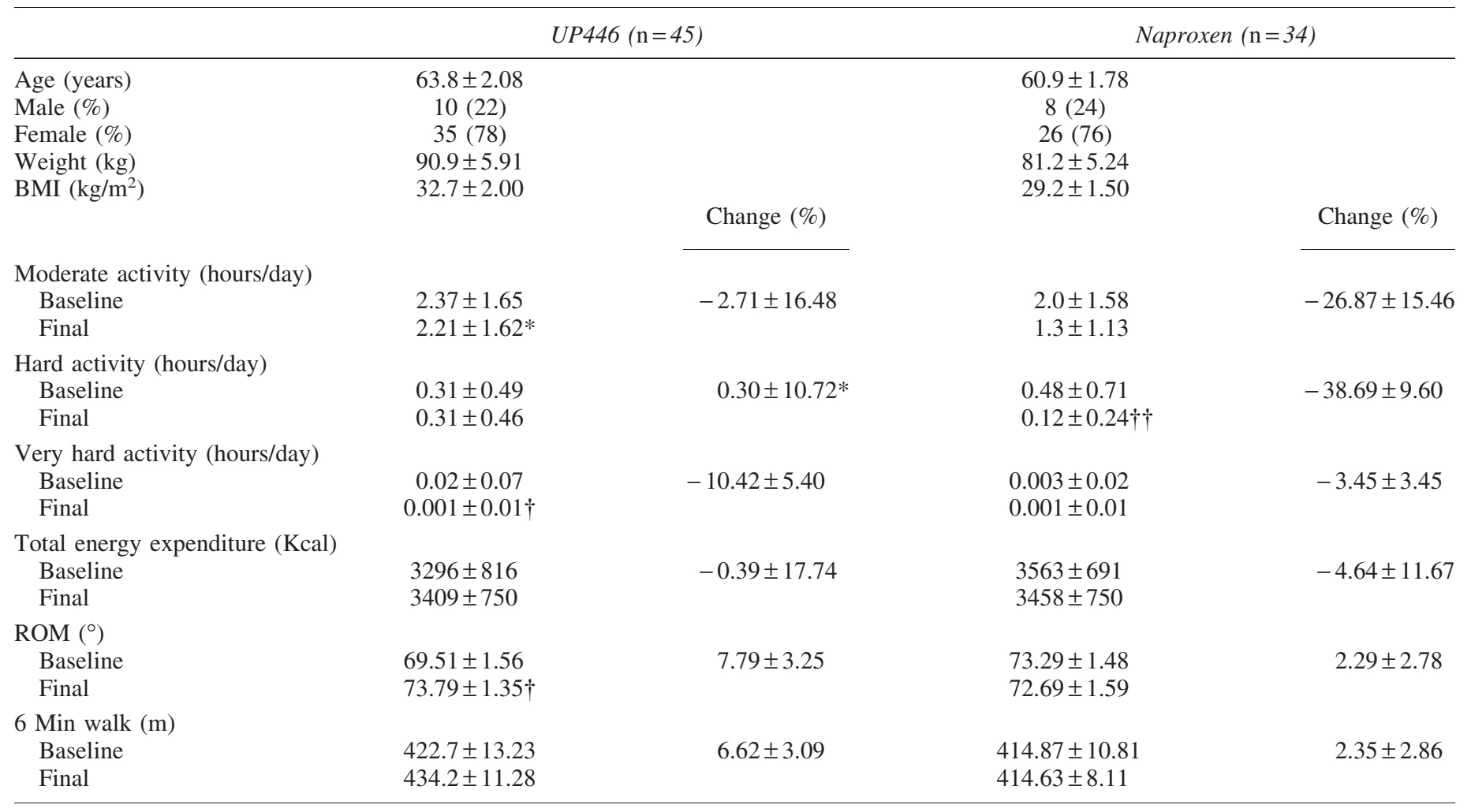

$* P \leq .05$ comparison between baseline and final values between groups. Mean \pm standard error $(S E) ; n=79 . \dagger P \leq .05$; $\dagger \uparrow P \leq .001$ comparison between baseline and final time points within each group. The mean $\pm S E$ of percent changes is shown in the Change column. 


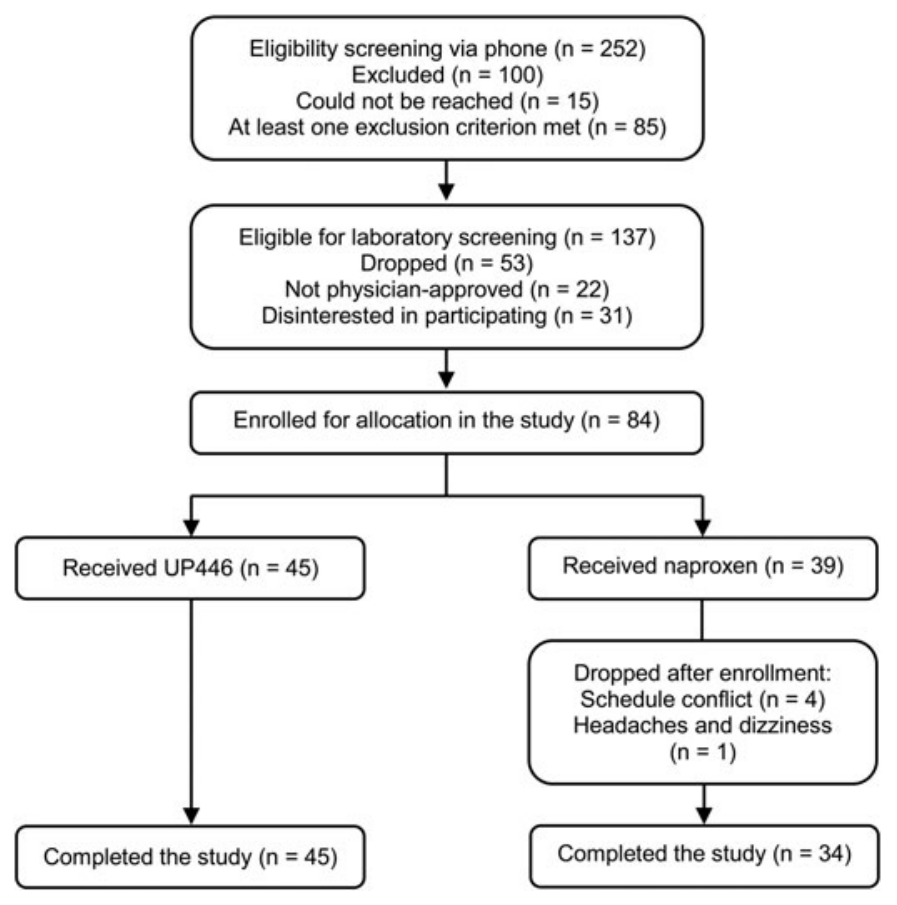

FIG. 1. Flowchart of recruitment and enrollment process.

\section{Pain, stiffness, and physical function}

WOMAC pain scale results are presented in Table 1 as well as in Figures $2-4$. UP446 significantly $(P=.009)$ reduced pain (Fig. 2) over the 1 week period, but no difference was observed between groups. Both groups had significant $(P=.0021$ UP446, $P=.0081$ naproxen) reductions in stiffness from baseline to final (Fig. 3). Neither group experienced improvements in physical function during the 7 day period (Fig. 4).

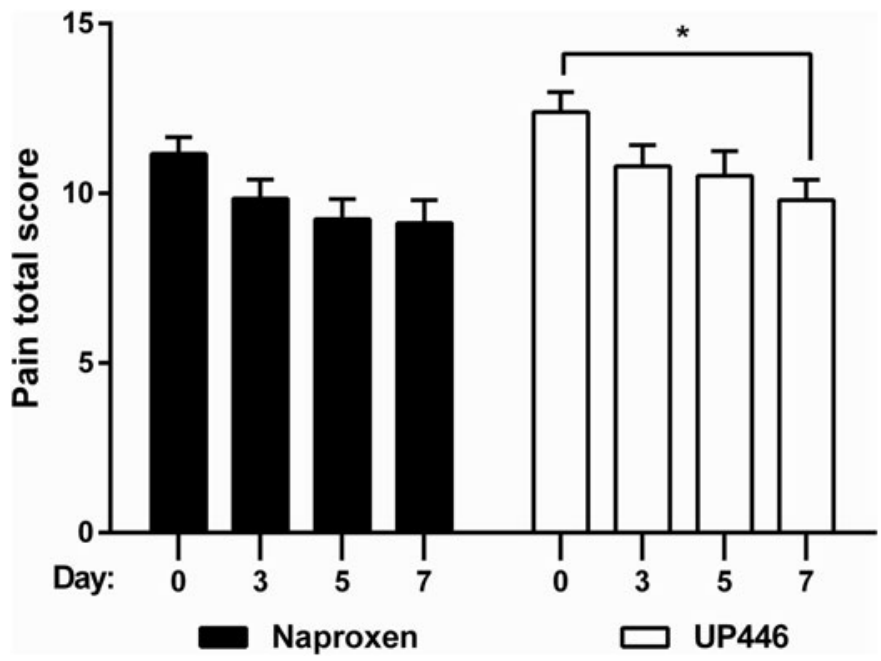

FIG. 2. UP446 efficacy for reducing pain across three time points and between groups, as represented by the total Western Ontario and McMaster Universities Arthritis Index (WOMAC) scores for pain. $* P \leq .05$ for the analysis of variance (ANOVA) comparison between baseline and final time points.

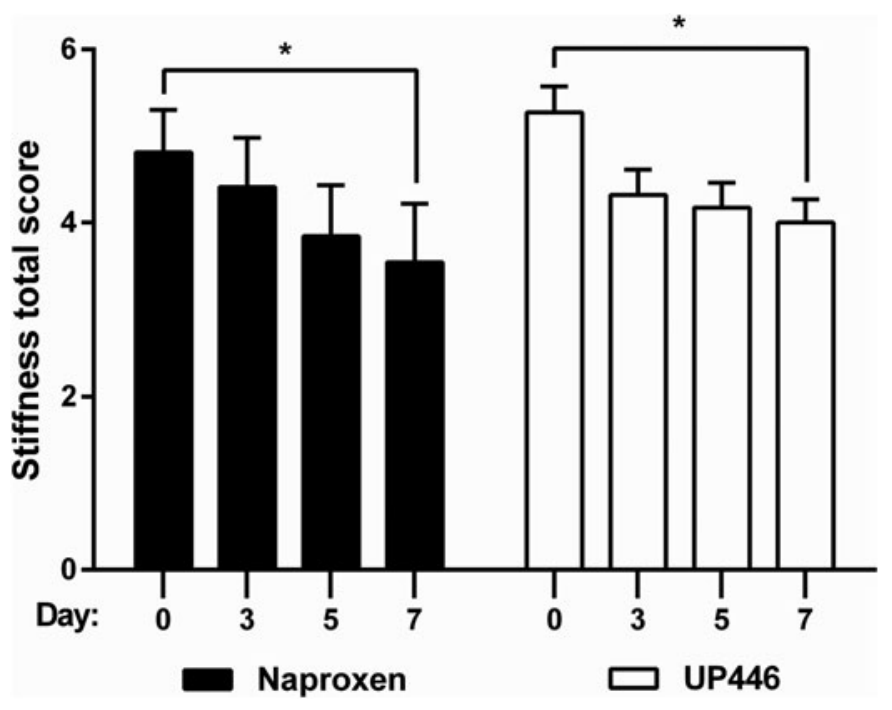

FIG. 3. Comparative effiacy of UP446 for attenuation of knee stiffness, represented by WOMAC scores. $* P \leq .05$ for the ANOVA comparison between baseline and final time points.

\section{Range of motion and walking function}

The effects of UP446 supplementation on ROM testing and 6MWT durations are shown in Table 1. No group differences were found for either variable. UP446 supplementation did significantly $(P=.036)$ improve ROM after the 1 week period, indicating a $6 \%$ increase for the UP446 group and $0.1 \%$ decrease in the naproxen group. No significance was observed for ROM over time within the naproxen group, which may suggest that the UP446 supplement provides an advanced aid in joint flexibility over the course of 1 week. No significant time interactions were observed with respect to the 6MWT.

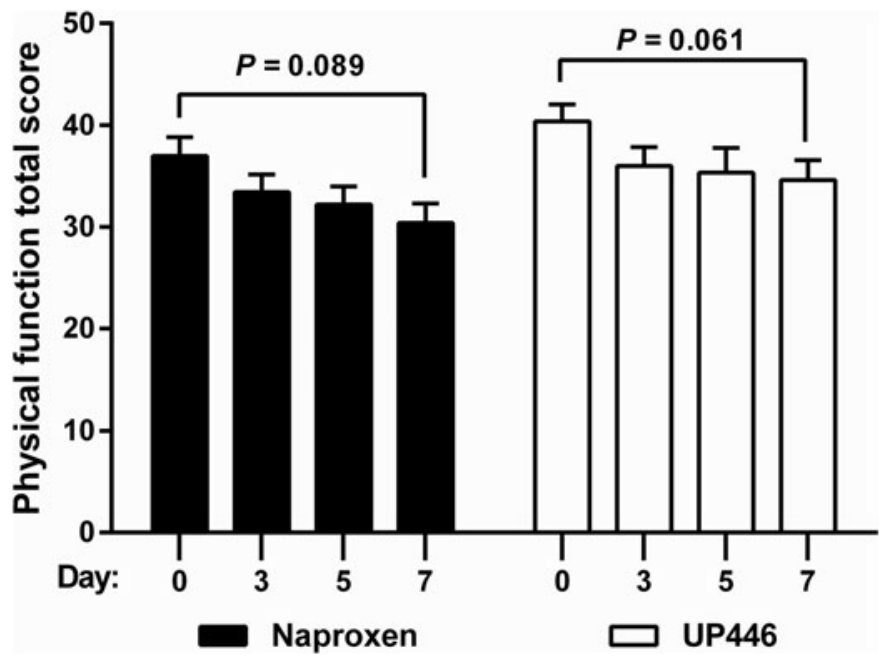

FIG. 4. Summary of WOMAC scores for physical function across three time points. No statistical significance was found in the ANOVA comparison. 


\section{Physical activity levels and energy expenditure}

Mean baseline activity scores were not statistically significant between groups. The raw mean moderate activity scores at final were statistically greater for the experimental group. However, both values decreased from baseline to final. The moderate activity score mean difference at final $(0.16 \pm 0.03$; $P=.63)$ for the UP446 group was less than that of the naproxen group $(0.7 \pm 0.45 ; P=.07)$. In the naproxen group, the amount of hard activity reported (Table 1 ) after the 1 week period was significantly decreased $(P<.001)$. In contrast, hours spent performing hard activities in the UP446 group remained the same. Mean very hard activity scores were reduced from baseline to final for both groups, but those receiving UP446 reported significantly lower activity $(P<.02)$. There were no statistical differences in total energy expenditure between groups at any time point (Table 1).

\section{Blood biomarkers of inflammation}

All reported data are shown in Table 2. No statistically significant group or time differences were indicated in serum concentrations of IL-6, CRP, or HA. Though not statistically significant, HA concentrations decreased from baseline for both groups ( $P=.18 \mathrm{UP} 446, P=.28$ naproxen). Interleukin- $1 \beta$ concentrations were significantly $(P=.02)$ higher in the UP446 treatment group compared to naproxen at final. No time interaction in IL-6 concentrations was found for either group. TNF- $\alpha$ concentrations increased significantly with both treatments $(P=.0007$ UP446, $P=.02$ naproxen).

\section{DISCUSSION}

Our results demonstrated that UP446-a natural dietary supplement-is capable of reducing knee OA-associated pain and improving knee joint flexibility after a 1 week period. The use of complementary and alternative medicine in order to treat knee OA is quite common, with many individuals using multiple supplements. ${ }^{24}$ Marketed dietary supplements and plant extracts are in high use for relieving symptomatic knee OA. Individuals with knee OA commonly take these supplements for an extended time, ${ }^{25}$ normally exceeding 1 month. Thus, it would be desirable to find compounds with shorter efficacy periods such as 1 week. The first study to exhibit the effects of UP446 in relieving
OA symptoms was carried out over a 90 day period. ${ }^{26}$ Sampalis and Brownell demonstrated that up to $500 \mathrm{mg} /$ day of UP446 dose-dependently improves WOMAC pain, stiffness, and functional impairment scores as soon as 30 days when compared to $200 \mathrm{mg} / \mathrm{day}$ of celecoxib. Thus, along with the present study, UP446 has been shown to be efficacious in a rapid manner and may somewhat exceed the effect of certain NSAIDs. Levy et al. ${ }^{16}$ reported the benefits of $S$. baicalensis and A. catechu extracts in individuals suffering from knee OA with extended use. In their study, time spent walking to cover a set distance was also significantly reduced to a greater extent than naproxen after 6 and 12 weeks of supplementation. These findings provided the foundation for the current research for which we report the beneficial effects of a shorter period of supplementation of a similar formulation. Levy et al. also determined differences in the level of OA severity (mild, moderate, or severe), suggesting that those who were older and affected more mildly may respond better to supplementation. In the current study, severity differences could not be analyzed and should be considered a limitation of the study. However, it is likely that many of the subjects were mild or moderate cases, which may explain the rapid response seen in both groups.

At the end of the 1 week study period, subjects on UP446 continued with the same level of activity as assessed by selfreported time spent performing moderate activities, such as a brisk walk (3-4 mph), and hard activities. While the ability to perform similar tasks was unaffected for subjects taking UP446, those who received naproxen experienced substantially lower activity of approximately fourfold. These results may suggest the superiority of UP446 over naproxen, which is commonly prescribed for patients with inflammatory conditions such as OA. However, we cannot offer an explanation for this observation. It is quite contrary to one's expectation; since both agents reduced pain, they should have also enhanced the ability of the subjects to endure more rigorous activities.

Based on WOMAC scores of the current study, pain was significantly attenuated following UP446 treatment. Naproxen also attenuated pain, albeit to a lesser degree, as represented by a plateau on day 7 . Numerically speaking, the steeper decline in scores after 3 days gives way to the indication of a rapid effect that continues, though at a lesser magnitude. Similar results were found in a 4 week study in which naproxen, flavocoxid, ${ }^{17}$ and UP446 ${ }^{20}$ (500 mg twice

Table 2. Effect of UP446 Supplementation on Serum Inflammatory Markers: Group and Time Interactions

\begin{tabular}{|c|c|c|c|c|c|c|}
\hline & \multicolumn{3}{|c|}{$U P 446(\mathrm{n}=45)$} & \multicolumn{3}{|c|}{ Naproxen $(\mathrm{n}=34)$} \\
\hline & Baseline & Final & Change (\%) & Baseline & Final & Change (\%) \\
\hline $\mathrm{IL}-1 \beta(\mathrm{pg} / \mathrm{mL})$ & $0.64 \pm 0.14$ & $0.73 \pm 0.14 *$ & $58.74 \pm 18.74$ & $1.14 \pm 0.35$ & $0.48 \pm 0.05$ & $31.12 \pm 13.77$ \\
\hline IL-6 (pg/mL) & $5.59 \pm 1.21$ & $4.09 \pm 0.77$ & $6.84 \pm 14.66$ & $2.76 \pm 0.26$ & $2.47 \pm 0.23$ & $-0.23 \pm 6.68$ \\
\hline TNF- $\alpha(\mathrm{pg} / \mathrm{mL})$ & $0.78 \pm 0.03$ & $1.05 \pm 0.07 \dagger \dagger$ & $43.18 \pm 13.03$ & $0.87 \pm 0.06$ & $1.07 \pm 0.07 \dagger$ & $35.88 \pm 12.15$ \\
\hline $\mathrm{CRP}(\mathrm{mg} / \mathrm{L})$ & $3.06 \pm 2.84$ & $3.11 \pm 3.07$ & $14.23 \pm 0.89 *$ & $2.22 \pm 1.96$ & $2.02 \pm 2.36$ & $-19.20 \pm 0.41$ \\
\hline HA (ng/mL) & $248 \pm 187.12$ & $198 \pm 107.04$ & $-0.78 \pm 0.48$ & $288 \pm 187.30$ & $241.85 \pm 178.86$ & $-11.76 \pm 0.45$ \\
\hline
\end{tabular}

Mean \pm SE; $n=79$. $* P \leq .05$ for the comparison between groups. $\uparrow P \leq .05$; $\uparrow P \leq .001$ for the comparison between baseline and final time points. 
daily) significantly reduced pain scale scores and selfreported discomfort among older men and women with knee OA. Thus, the available flavonoids were equally as potent as the often-used NSAID. In the 3 month study of pycnogenol $(150 \mathrm{mg} /$ day $)$, a potent flavonoid-rich dietary extract, significant decreases in pain and improved physical function in comparison to the nonanalgesic placebo were indicated. ${ }^{27}$ Though they observed no statistical differences between groups with regard to stiffness, an improvement was noted from baseline to final in those receiving the supplement. Our findings are in agreement, as we report notable alterations in stiffness among both groups after only 1 week.

Research personnel did their best to stress the difference between the feelings of pain and stiffness for greater accuracy. Yet it is likely that several subjects could not effectively report stiffness without the influence of pain. Thus, the quantitative measure of ROM was critical for assessing improvements in the function of the knee. We report a substantial enhancement in ROM of subjects with mild to moderate knee $\mathrm{OA}$ - an effect not seen in those receiving naproxen. In another investigation, the consumption of acai pulp (Euterpe oleracea) slightly improved $(P<.06)$ ROM in middle-age to elderly subjects with mild to moderate joint pain after 12 weeks. ${ }^{28}$ Improvements in the ability to walk were observed despite a slight increase in distance covered in the UP446 group. This, at least in part, may be explained by the inability to control for the effort put forth by each subject. We were unable to control for variables such as tiredness or lack of motivation, which undoubtedly influenced subject effort during testing.

Our intent was not to recruit overweight subjects for this study. Yet all BMI values were in the overweight to obese range $\left(30.5 \pm 1.8 \mathrm{~kg} / \mathrm{m}^{2}\right.$ for both groups). This observation supports the notion that $\mathrm{OA}$ and obesity are linked. Numerous studies have reported such an outcome, ${ }^{1,29-32}$ further supporting the relationship. Obesity is considered a major risk factor for knee OA, substantially reduces quality of life, ${ }^{33}$ and may contribute to its development via several ways, including excessive loading exerted on knee articular cartilage and highly proinflammatory microenvironment that lead to cartilage degradation. ${ }^{26}$ Therefore, it may be advantageous to combine the use of UP446 with an exercise and diet regimen.

Proinflammatory molecules that are responsible for cellular recruitment for cartilage degradation and chondrocyte apoptosis ${ }^{34}$ play an important role in understanding the activity of S. baicalensis and A. catechu in the current study. Older individuals with osteoarthritis tend to have high systemic CRP, TNF- $\alpha$, IL- 6 , and IL- $1 \beta$ concentrations by default. ${ }^{31,35}$ Among the circulating inflammatory molecule concentrations measured in the present study, all but TNF- $\alpha$ remained statistically unchanged in both groups. These increases in TNF- $\alpha$ were not expected. However, it is not uncommon to observe elevated concentrations, especially in those with mid-stage to severe osteoarthritis. High circulating levels of TNF- $\alpha$ and IL- 6 tend to be associated with lower physical function and slower walking speed in the elderly with knee $\mathrm{OA},{ }^{31,36}$ but in the present study, this was not the case. Because TNF- $\alpha$ plays such a pivot role in the conservation of $\mathrm{OA}$ and is associated with greater radiographic of knee occurrences, ${ }^{35} 1$ week of treatment is unlikely a long enough duration to observe decreases, especially considering that many of the subjects were inactive prior to the 6MWT. This outcome does not detract from the positive effect indicated by functional and qualitative testing, but supports the need for longer treatment and mechanistic study of UP446. Serum HA indicates synovial turnover ${ }^{37}$ and we found somewhat of a reduction in HA for both groups. Such a result may support the notion of local effects. However, the current data cannot justify this.

In summary, the results of this study suggest that, similar to that of naproxen, UP446 is capable of reducing perceived joint pain and stiffness while also improving joint flexibility. Our findings also provide additional evidence for the analgesic effects of plant extracts $S$. baicalensis and A. catechu. Whether or not the supplement offers improvements in mobility will require further investigation. Future studies should include multiple doses of UP446 with the addition of a placebo group, and duration of study of up to 3 months with periodic measures. By implementing this model, researchers would be able to pinpoint further the amount of time needed to see significant changes, if any, in both mobility and cytokine measurements.

\section{ACKNOWLEDGMENTS}

This research project was funded by Unigen, Inc. (Seattle, WA, USA). While the company was involved during the testing process and made aware of all protocols used, the performance of the study and the preparation and submission of this manuscript were not influenced by the company. The authors wish to thank Wyatt Eddy, Rafaela G. Feresin, Yelizaveta Muravyeva, and Zahra Ezzat-Zadeh for their substantial contributions during the testing and recruitment phases of the study.

\section{AUTHOR DISCLOSURE STATEMENT}

No competing financial interests exist.

\section{REFERENCES}

1. Felson DT, Gross KD, Nevitt MC, et al.: The effects of impaired joint position sense on the development and progression of pain and structural damage in knee osteoarthritis. Arthritis Rheum 2009;61:1070-1076.

2. Zochling J, March LM, Lapsley H, et al.: Use of complementary medicines for osteoarthritis-A prospective study. Ann Rheum Dis 2004;63:549-554.

3. Bruyere O, Reginster JY: Glucosamine and chondroitin sulfate as therapeutic agents for knee and hip osteoarthritis. Drug Aging 2007;24:573-580.

4. Bassleer CT, Franchimont PP, Henrotin YE, et al:: Effects of ipriflavone and its metabolites on human articular chondrocytes cultivated in clusters. Osteoarthr Cartilage 1996;4:1-8.

5. Blotman F, Maheu E, Wulwik A, et al.: Efficacy and safety of avocado/soybean unsaponifiables in the treatment of symptomatic osteoarthritis of the knee and hip. A prospective, multicenter, 
three-month, randomized, double-blind, placebo-controlled trial. Rev Rhum Engl Ed 1997;64:825-834.

6. Manthey JA: Biological properties of flavonoids pertaining to inflammation. Microcirculation 2000;7:S29-S34.

7. Maroney MA, Alcaraz MJ, Forder RA, et al.: Selectivity of neutrophil 5-lipooxygenase and cyclooxygenase inhibition by an anti-inflammatory glycoside and related aglycone flavonoids. J Pharm Pharmacol 1988;40:787-792.

8. Altavilla D, Squadrito F, Bitto A, et al.: Flavocoxid, a dual inhibitor of cyclooxygenase and 5-lipoxygenase, blunts proinflammatory phenotype activation in endotoxin-stimulated macrophages. Br J Pharmacol 2009;157:1410-1418.

9. Lin N, Sato T, Takayama Y, et al.: Novel anti-inflammatory actions of nobiletin, a citrus polymethoxy flavonoid, on human synovial fibroblasts and mouse macrophages. Biochem Pharmacol 2003;65:2065-2071.

10. Hawkey CJ: COX-2 inhibitors. Lancet 1999;353:307-314.

11. Lazzaroni M, Porro GB: Gastrointestinal side-effects of traditional non-steroidal anti-inflammatory drugs and new formulations. Aliment Pharmacol Ther 2004;20:48-58.

12. Bensen WG, Fiechtner JJ, McMillen JI, et al.: Treatment of osteoarthritis with celecoxib, a cyclooxygenase-2 inhibitor: a randomized controlled trial. Mayo Clinic Proc 1999;74:1095-1105.

13. Essex MN, Bhadra P, Sands GH: Efficacy and tolerability of celecoxib versus naproxen in patients with osteoarthritis of the knee: a randomized, double-blind, double-dummy trial. J Int Med Res 2012;40:1357-1370.

14. Kuptniratsaikul V, Pinthong T, Bunjob M, et al.: Effiacy and safety of Derris scandens benth extracts in patients with knee osteoarthritis. J Altern Compleme Med 2011;17:147-153.

15. Burnett BP, Jia Q, Zhao Y, et al.: A medicinal extract of Scultellaria baicalensis and Acacia catechu acts as a dual inhibitor of cyclooxygenase and 5-lipoxygenase to reduce inflammation. J Med Food 2007;10:442-451.

16. Levy R, Khokhlov A, Kopenkin S, et al.: Efficacy and safety of flavocoxid compared with naproxen in subjects with osteoarthritis of the knee-A subset analysis. Adv Ther 2010;27:953-962.

17. Levy RM, Saikovsky R, Shmidt E, et al:: Flavocoxid is as effective as naproxen for managing the signs and symptoms of osteoarthritis of the knee in humans: A short-term randomized, double-blind pilot study. Nutr Res 2009;29:298-304.

18. Yimam M, Brownell LA, Pantier M, Jia Q: UP446, analgesic and anti-inflammatory botanical composition. Pharmacognosy Res 2013;5:139-145.

19. Lee YC, Hyun E, Yimam M, Brownell L, Jia Q: Acute and 26week repeated oral dose toxicity study of UP446, a combination of Scutellaria extract and Acacia extract in rats. Food Nutr Sci 2013;4:14-27.

20. Sampalis JS, Brownell LA: A randomized, double blind, placebo and active comparator controlled pilot study of UP446, a novel dual pathway inhibitor anti-inflammatory agent of botanical origin. Nutr J 2012;11:21.

21. Bellamy N, Buchanan WW, Goldsmith $\mathrm{CH}$, et al:: Validation study of WOMAC: a health status instrument for measuring clinically important patient relevant outcomes to antirheumatic drug therapy in patients with osteoarthritis of the hip or knee. $J$ Rheumatol 1988;15:1833-1840.

22. Sallis JF, Haskell WL, Wood PD, et al.: Physical activity assessment methodology in the five-city project. Am J Epidemiol 1985;121:91-106.

23. Guyatt GH, Sullivan MJ, Thompson PJ, et al.: The 6-minute walk: A new measure of exercise capacity in patients with chronic heart failure. Can Med Assoc J 1985;132:919-923.

24. Lapane KL, Sands MR, Yang S, et al.: Use of complementary and alternative medicine among patients with radiographicconfirmed knee osteoarthritis. Osteoarthr Cartil 2012;20:22-28.

25. Akhtar N, Haqqi TM: Current nutraceuticals in the management of osteoarthritis: a review. Ther Adv Musculoskeletal Dis 2012;4: 167-180.

26. Lee R, Kean WF: Obesity and knee osteoarthritis. Inflammopharmacol 2012;20:53-58.

27. Farid R, Mirfeizi Z, Mirheidari M, et al:: Pycnogenol supplementation reduces pain and stiffness and improves physical function in adults with knee osteoarthritis. Nutr Res 2007;27:692-697.

28. Jensen GS, Ager DM, Redman KA, et al.: Pain reduction and improvement in range of motion after daily consumption of an acai (Euterpe oleracea Mart.) pulp-fortified polyphenolic-rich fruit and berry juice blend. J Med Food 2011;14:702-711.

29. Arjmandi BH, Khalil DA, Lucas EA, et al:: Soy protein may alleviate osteoarthritis symptoms. Phytomedicine 2004;11:567575.

30. Clegg DO, Reda DJ, Harris CL, et al.: Glucosamine, chondroitin sulfate, and the two in combination for painful knee osteoarthritis. N Engl J Med 2006;354:795-808.

31. Penninx BW, Abbas H, Ambrosius W, et al:: Inflammatory markers and physical function among older adults with knee osteoarthritis. J Rheumatol 2004;10:2027-2031.

32. Rousseau JC, Delmas PD: Biological markers in osteoarthritis. Nat Clin Pract Rheumatol 2007;3:346-356.

33. Losina E, Walensky RP, Reichmann WM, et al:: Impact of obesity and knee osteoarthritis on morbidity and mortality in older Americans. Ann Intern Med 2011;154:217-226.

34. López-Armada MJ, Caramés B, Lires-Deán M, et al.: Cytokines, tumor necrosis factor-alpha and interleukin-1beta, differentially regulate apoptosis in osteoarthritis cultured human chondrocytes. Osteoarthr Cartil 2006;14:660-669.

35. Stannus O, Jones G, Cicuttini F, et al.: Circulating levels of IL-6 and TNF- $\alpha$ are associated with knee radiographic osteoarthritis and knee cartilage loss in older adults. Osteoarthr Cartil 2010;18:1441-1447.

36. Miller GD, Nicklas BJ, Loeser, RF: Inflammatory biomarkers and physical function in older, obese adults with knee pain and self-reported osteoarthritis after intensive weight-loss therapy. JAGS 2008;56:644-651.

37. Belo JN, Berger MY, Reijman M, Koes BW, Bierma-Zeinstra SMA: Prognostic factors of progression of osteoarthritis of the knee: a systematic review of observational studies. Arthritis Rheum 2007;57:13-26. 